\title{
Clinical Profile of Multiple Gestations at a Tertiary Care Hospital
}

\author{
Jeevan S Nair ${ }^{1}$ \\ ${ }^{1}$ Assistant Professor, Department of Paediatrics, Mount Zion Medical College, Chayalode, Ezhamkulam, Adoor.
}

\section{Abstract}

Background: Monochorionic twins showed increased incidence of discordant growth as compared to dichorionic twins. Studies have reported fivefold mortality of twins in comparison with singleton pregnancy. In this a prospective study is done to assess the perinatal, neonatal mortality and morbidity in multiple gestations. Zygosity determines the degree of risk of chromosomal abnormalities in each fetus of a multiple gestation. The risk for aneuploidy in each fetus of an MZ pregnancy is the same as a singleton pregnancy, and except for rare cases of genetic discordancy, both fetuses are affected. In a DZ pregnancy, each twin has an independent risk for aneuploidy, and therefore has twice the risk of having a chromosomal abnormality compared with a singleton. Subjects and Methods: All mothers with multiple gestation were recruited to this study. Twins and Triplets in OBG ie. new born brought from other hospitals were excluded. Statistical analysis was done as percentage. Results: 80 twins were studied, out of which preterm deliveries constituted $87.5 \%$ of all deliveries. Most of the mothers were between 25-29 year age group and most of them were primi. There was family history of twining in $10 \%$ of the study group. Conclusion: Vaginal delivery was done for $68 \%$ of twins and LSCS for $23.8 \%$.

Keywords: Multiple Gestations, Zygosity, Chromosomal Abnormality.

Corresponding Author: Dr. Jeevan S Nair, Assistant Professor, Department of Paediatrics, Mount Zion Medical College, Chayalode, Ezhamkulam, Adoor.

Received: January 2019

Accepted: January 2019

\section{Introduction}

Multiple gestation is a high risk pregnancy. It is associated with increased risk of morbidity and mortality for fetus and neonates. Twin gestation is responsible for $10 \%$ of all perinatal. There is increased incidence of LBW and prematurity in such neonates. Antecedent complications in mother like preeclampsia tend to increase risk in twin neonates. LBW is a most important factor correlating with mortality rates. Discordant infants and triplets have higher risk for cognitive delays in the first two years of life. Monochorionic twins showed increased incidence of discordant growth as compared to dichorionic twins. Studies have reported fivefold mortality of twins in comparison with singleton pregnancy. In this a prospective study is done to assess the perinatal, neonatal mortality and morbidity in multiple gestation. ${ }^{[1]}$

Two main account for the increase in multiple births over the last two decades: one factor is the increasing use of ovulation-inducing drugs and intrauterine insemination and artificial reproductive technologies (ARTs) such as in vitro fertilization (IVF), although the number of ART procedures involving the transfer of three or more embryos has declined between 1997 and 200. A second factor is the trend toward older maternal age at childbearing (peak at 35-39 years), which is associated with an increase in multiples. ${ }^{[2]}$
MZ pregnancies result from the splitting of a single egg anywhere from day 0 to day 14 post fertilization, and therefore a spectrum of placentas can form depending on the day of embryo splitting.

A dichorionic diamniotic placentas can result when early splitting occurs at day 0 to day 3 before chorion formation (which usually occurs around day 3) and before implantation. A monochorionic diamniotic placenta results if splitting occurs around day 4 to day 7 , at which time the blastocyst cavity has developed and the chorion has formed. Amnion formation occurs at day 6 to day 8, and splitting, of the egg after this time (day 4-7) can result in a monochorionic monoamniotic placenta. At day 14 and thereafter, the primitive streak begins to form and late splitting of the embryo at this time results in conjoined twins. ${ }^{[3]}$

DZ or multizygous pregnancies result when mote than one dominant follicle has matured during the same menstrual cycle and multiple ovulations occur. Increased levels of the follicle stimulating hormone (FSH) in the mother have been associated with spontaneous DZ twinning. FSH levels increase with advanced maternal age (peak at age -37). A familial tendency towards twinning has also been shown to be associated with increased levels of FSH.

Multiple gestational sacs can be detected by ultrasonography as early as 5 weeks and cardiac activity can be detected from 


\section{Nair; Clinical Profile of Multiple Gestations}

more than one fetus at 6 weeks. First trimester ultrasonography can best determine the chorionicity of a multiple gestation; chorionicity is more difficult to determine in the second trimester. From week 10 to week 14 , a fused dichorionic placenta may often be distinguished from a true monochorionic placenta by the presence of an internal dividing membrane or ridge at the placental surface (lambda sign). The dividing septum of a dichorinic placenta appears thicker and includes two amnions and two chorionic layers. In contrast, the dividing septum of amniochorionic placenta consists of two thin amnions. One placenta, samesex fetuses, and absence of a dividing septum suggests monoamniotic twins, but absence of a dividing septum may also be due to septal disruption. Both conditions have a poor prognosis. ${ }^{[4,5]}$

\section{Subjects and Methods}

All mothers with multiple gestation were recruited to this study

1) Mother details taken Histroy of any drug intake or assisted reproductive techniques are also taken.

2) Findings in USG are followed up.

3) Cord blood for hemoglobin PVC and blood grouping are sent.

4) After delivery placenta is examined to know about chrorinicity.

5) Immediate outcome is assessed in neonates such as

6) Birth weight

7) Chromosomal anomalies

8) Deformations

\section{Inclusion criteria}

All multiple deliverse in Hospital

\section{Exclusion Criteria}

Twins and Triplets in OBG ie. new borns brought from other hospitals.

\section{Results}

Table 1: Percentage Distribution of the Sample According to Maternal Age

\begin{tabular}{|l|l|l|}
\hline Maternal age & Count & Percentage \\
\hline $20-24$ & 26 & 32.5 \\
\hline $25-29$ & 32 & 40.0 \\
\hline $30+$ & 22 & 27.5 \\
\hline
\end{tabular}

Table 2: Percentage Distribution of the Sample According To Gravida

\begin{tabular}{|l|l|l|}
\hline Gravida & Count & Percentage \\
\hline Primi & 45 & 56.3 \\
\hline G2 & 25 & 31.3 \\
\hline G3 and more & 10 & 12.5 \\
\hline
\end{tabular}

Table 3: Percentage Distribution of the Sample According To Family

\begin{tabular}{|l|l|l|}
\hline Family History & Count & Percentage \\
\hline Yes & 10 & 12.5 \\
\hline No & 70 & 87.5 \\
\hline
\end{tabular}

Table 4: Percentage Distribution of the Sample According to the Use of Assisted Reproductive Technique

\begin{tabular}{|l|l|l|}
\hline $\begin{array}{l}\text { Assisted Reproductive } \\
\text { technique }\end{array}$ & Count & Percentage \\
\hline Yes & 10 & 12.5 \\
\hline No & 70 & 87.5 \\
\hline
\end{tabular}

Table 5: Percentage Distribution of the Sample According to the Use of Assisted Reproductive Technique

\begin{tabular}{|l|l|l|}
\hline $\begin{array}{l}\text { Assisted Reproductive } \\
\text { technique }\end{array}$ & Count & Percentage \\
\hline Clomiphene & 9 & 90.0 \\
\hline IVF & 1 & 10.0 \\
\hline
\end{tabular}

Table 6: Percentage Distribution of the Sample According to Placenta

\begin{tabular}{|l|c|l|}
\hline Placenta & Count & Percentage \\
\hline MCMA & 5 & 6.3 \\
\hline MCDA & 7 & 8.8 \\
\hline DCDA & 65 & 81.3 \\
\hline DCTA & 3 & 3.8 \\
\hline
\end{tabular}

Table 7: Percentage Distribution of the Sample According to Number of Fetus

\begin{tabular}{|l|l|l|}
\hline No. of Fetus & Count & Percentage \\
\hline Twins & 77 & 96.3 \\
\hline Triplets & 3 & 3.7 \\
\hline
\end{tabular}

- 80 twins were studied, out of which preterm deliveries constituted $87.5 \%$ of all deliveries.

- Most of the mothers were between 25-29 year age group and most of them were primi.

- There was family history of twining in $10 \%$ of the study group.

- Assisted reproductive techniques were used by $12.5 \%$ of couples ad $90 \%$ of them used clomiphene cirate and $10 \%$ used in vitro fertilization.

- Dichorionic twins accounted for $85 \%$ of the total multiple deliveries.

- Vaginal delivery was done for $68 \%$ of twins and LSCS for $23.8 \%$.

- TTS was found I 2 gestations out of which one baby required transfusion.

- Anomalies was found in 2 babies one major - Anorectal Malformation one minor - polydactyly.

- SGA babies constituted $55.8 \%$ of the study group.

\section{Discussion}

Monozygotic (MZ) twins originate and develop from a single fertilized egg (zygote) as a result of division of the inner cell mass of the blastocyst. MZ twins are of the same sex and are genetically identical. Dizyotic (DZ) or fraternal twins originate and develop from two separated fertilized eggs. Triples and higher-order pregnancies (quadruplets, quintuplets, sextuplets,septuplets, etc.) Can be mutizygotic, $\mathrm{MZ}$ and identical, or rarely, a combination of both.

A major portion of the placenta and the fetal membranes originate from the zygote. The placenta consists of two 
parts: (1) a larger fetal part derived from the villous chorion and (2) a smaller maternal part derived from the deciduas basalis. The chorionic and amniotic sacs surround the fetus. The chorion to from at day 3 after fertilization, and the amnion begins to form between day 6 and day 8 . The two membranes eventually fuse to form the amniochorionic membrane.

MZ twins commonly have one placenta with one chorion and two amnions (monochorionic diamniotic) or rarely, one placenta with one chorion and one amnion (monochorionic monoamniotic). If early splitting occurs before the formation of the chorion and amnion (day 0-3), MZ twins can end up having two placentas with two chorions and two amnions (dichorionic diamniotic). DZ twins always have two placentas with two chorions and two amnions (dichorionic diamniotic); however the two placentas and chorions may be fused. $^{[6]}$

The rate of $\mathrm{MZ}$ twinning has remained relatively constant (3.5 per 1,000). The rate of DZ twinning is approximately 1 in 100 births. This rare is influenced by several factor such as ethnicity ( 1 in 500 Asians, 1 in 125 in whites, and as high as 1 in 20 in Afeican populations) and maternal age. The frequency of DZ twinning has a genetic tendency that is a affected by the genotype of the mother and nor that of the father.

Deoxyribonucleeic acid (DNA) typing can be used to determine zygosity in same-sex twins. Prenatally, can be obtained by chorionic villus sampling (CVS) or amnoiocentesis. Postnatally, DNA typing should optimally be performed on umbilical cord tissue buccal smear, or a skin biopsy specimen instead of blood. There is evidence that DZ twins, even in the absence of vascular connections, can also carry hematopoietic stem cells (HSCs) derived from their twin. HSCs are most likely transferred from one fetus to the other through maternal circulation. ${ }^{[7]}$

Zygosity determines the degree of risk of chromosomal abnormalities in each fetus of a multiple gestation. The risk for aneeuploidy in each fetus of an $\mathrm{MZ}$ pregnancy is the same as a singleton pregnancy, and except for rare cases of genetic discordancy, both fetuses are affected. In a DZ pregnancy, each twin has an independent risk for aneuploidy, and therefore has twice the risk of having a chromosomal abnormality compared with a singleton. ${ }^{[8]}$

For women with multiples is limited because each fetus contributes variable levels of these serum markers. When levels are abnormal, there is difficulty in identifying which fetus is affected. To assess for nuchal translucency is a more sensitive and specific test to screen for chromosomal abnormalities. A second trimester ultrasonography exam is important in surveying each fetus for anatomic defects. Second-trimester amniocentesis and first-trimester CVS can be safely performed on multiple and are both accurate diagnostic procedures for determining aneuploidy. ${ }^{[9]}$

Twin pregnancies should be evaluated for anomalies by fetal ultrasonography or more invasive procedures if indicated.
Congenital anomalies are concordant only in a minority of cases, even in MZ twins. Whether assisted reproductive techniques result in an increased incidence in congenital births defects is uncertain. ${ }^{[10]}$

Chromosomal anomalies occur at a higher frequency in offspring of multiple gestation. Advanced maternal age contribute to the increased risk in chromosomal anomalies seen in multiple gestation. The risk in $\mathrm{MZ}$ twins is equivalent to that of a singleton. The risk in DZ twins is independent for each fetus, so the risk of chromosomal abnormality in at one DZ twin is twice that of a singleton fetus. ${ }^{[1]}$

Conjoined twins result when incomplete embryonic division occurs late after day 14 post conception. At this time, differentiation of the chorion and amnion has occurred, and therefore conjoined twins are seen only in monochorionic monoamniotic twins. Conjoined twins are rare and occur in approximately 1 in 50,000 to 100,000 births. The most common sites of fusion are the chest and / or abdomen. Survival is rare when there is cardiac or cerebral fusion. In one small case series of conjoined twins, $28 \%$ died in utero, $54 \%$ died immediately after birth, and only $18 \%$ survived. Serial ultrasonography can define the fetal anatomy and help determine management options. Polyhydramnios can affect as many $50 \%$ of cases of conjoined twins and may require amnioreduction. Elective cesarean delivery close to term is recommended, and in cases where in one twin is not likely to survive, delivery of the cot win by an ex utero intra partum treatment (EXIT) procedure should be considered. Surgical separation should be performed emergently in the event that one twin dies, and survival of the cot win in these cases is $30 \%$ to $50 \%$. Survival is $80 \%$ to $90 \%$ in twins that undergo elective separation, which is usually performed at 2 to 2 months of age.

The pathophysiology if TTTS is not completely understood, but anomalous placental vascular connections between twins necessary for it to occur. Eight-five superficial arterial-toarterial (AA) and venous-to-venous (V\T) anastomoses that are potentially bidirectional and deep inter fetal artery-tovein (AV) communications located in the placental cotyledons that are supplied by one fetus an drained by the other. AA connections are thought to be protective whereas $\mathrm{AV}$ anastomoses lead to shunting of blood from one twin to the other. TTTS results when the superficial network of anastomoses does not compensate for the interim AV shunts. Five percent to $10 \%$ of monochorionic placentas have sufficient circulatory imbalance to produce TTTS. One fetus (the donor) slowly bold into cotwin's circulation (the recipient). Complications in the donor include anemia, hypervolemia, growth restriction, brain ischemic lesions, renal insufficiency, oligohydramnios ("stuck twin"), lung hypoplasia, limb deformation, and high risk for fetal demise. Complications int the recipient include ploycythemia, thrombosis, cerebral emboli, disseminated intravascular coagulation (DIG), polyhydramnios, progressive 


\section{Nair; Clinical Prafile of Multiple Gestations}

cardiomyopathy due to volume overload, and fetal hydrops. Newer evidence suggests that the pathophysiology of TTTS involve changes in the rennin-angiotensin system and endpthlin-1. Vasoactive mediators produced in the donor are shunted to the recipient resulting in hypertension, contributing to the development of hypertensive cardiomyopathy.

Diagnosis is usuallymade between 17 and 26 weeks' gestation, but the process may occur as early as 13 weeks. Severe cases of TTTS have signs before 20 weeks' gestation and have a mortality of $60 \%$ to $100 \%$. Diagnostic criteria for TTTS include monochorionicity, pkolyhydramnios in the sac of one twin (the recipient) and oligohydramnios in the sac of the other twin (the donor), umbilical cord size discrepancy, cardiac dysfunction in the polyhydramniotic twin, abnormal umbilical artery and/ or ductus venosus Doppler velocimetry,and significant growth discordance $(>20 \%){ }^{[11]}$

Fetal treatment interventions include serial amnioreduction microseptostomy of the intertwine membrane, fetoscopic laser photocoagulation, and selective feticide. A randomized clinical trial comparing amnioreduction and septostomy showed that only. One procedure was required with septostomy whereas serial amnioreduction required multiple procedure. Results from the Eurofoetus trial serial found that laser photocoagulation improved both perinatal survival and short-term neurologic outcome at 6months of life compared with serial amnioreduction. However, the selection of patients most likely to benefit, the best intervention fpr a particular patient, and the optimal timing of intervention remain uncertain. In addition, data on long-term neurodevelopmental outcome in surviving infants of TTTS (i.e., the incidence of cerebral palsy [CPI) are needed. ${ }^{[12]}$

Neonatal management may include resuscitation at birth and need for continued ventilator and cardiovascular support; rapid establishment of intravascular access for volume expansion to treat hypotension, correct hypoglycemia, and transfuse " packed red blood cells" to treat anemia; partial exchange transfusion in the recipient to treat significant polycythemia; and neuroimaging to detect central nervous system (CNS) injury.

Velamentous cord insertion and vasa previa occur 6 to 8 times more often in twins than in singletons, and even more Often in higher-order gestation. Probable factors contributing to this higher. Risk includes placental crowding and abnormal blastocyst implementation; all types of
Placentation can be affected. With velamentous cord insertion, vessels are unprotected Wharton jelly and are more prone to compression, thrombosis, or disruption, leading to fetal distress or hemorrhage.

Cord blood flow interruption due to cord accidents (i.e., cord entanglement pr compression) accounts for the reported high rate of intrauterine death of one or both twins. The period of highest risk is 26 to 32 weeks. The overall perinatal mortality in monochorionic monoamniotic twins is reported to be in the range of $12 \%$ to $60 \%$. More recent literature reports lower rates of perinatal mortality due to umbilical cord accident. This reduction may be attribute to improved prenatal diagnosis of monochorionic monoamniotic pregnancies, closer fetal surveillance, and increased delivery of multiples by cesarean section.

\section{Conclusion}

- Most common type of delivery was vaginal delivery (68\%) followed by LSCS (23\%).

- Out of the variables studied, significant influence on neonatal mortality was seen only with discordant twins.

\section{References}

1. Silva PA, Crosado B. The growth and development of twins compared with singletos at ages 9 and 11. Aistr Ped J 1985; 21:265267.

2. Growth standards for twins from birth to four years. Ann Hum Biol 1974; 1: 175-188.

3. Salat-Baroux J, Bourdrez, Antoine JM. Multiple pregnancies: the price to pay. Eur J.obset Gynecol Reprod Biol. 1996;65 (suppl): S17S18.

4. Ziaden SM. The outcome of triplet versus twin pregnancies. Gynecol Obstet Invest. 2000;50:96-99

5. Feldman R, Eidelman Al, Intervention methods for premature infants: how and do they affect development? Clin Perinatol 1998;25;613-626

6. Wolke D. Psychological development of prematurely born children Arch Dis Child. 1998;78:567-570

7. Hack M, Faaroff AA. Outcomes of children of extremely low birth weight and gestational age in the 1990s. Semin Neonatol. 200;5 :9106

8. Blickstein I. Normal and abnormal growth of multiples. Semin Neonatol. 2002; 7: 177-185

9. Louhiala P. Risk indicators of mental retardation : changes between 1967 and 1981. Dev Med Child Neurol, 1995 37 : 631-636.

10. Watt J. Temperamen in small for dates ad preterm infants: a preliminary study. Child Psychiatry Hum Dev. 1987:17:177-188.

11. Garel M. Salobir, Lelong N, Blndel B. Development and hehaviour of 7 year old triplets.Acta Paediatr. 2001;90:53-543.

12. Bryan E. Twins, Triplets and More : Their Nature, Development, and Care London, United Kingdom: 1922

Copyright: (C) the author(s), publisher. Asian Journal of Clinical Pediatrics and Neonatology is an Official Publication of "Society for Health Care \& Research Development". It is an open-access article distributed under the terms of the Creative Commons Attribution NonCommercial License, which permits unrestricted non-commercial use, distribution, and reproduction in any medium, provided the original work is properly cited.

How to cite this article: Nair JS. Clinical Profile of Multiple Gestations at a Tertiary Care Hospital. Asian J. Clin. Pediatr. Neonatol.2018;6(4):1-4.

DOI: dx.doi.org/10.21276/ajcpn.2018.6.4.1

Source of Support: Nil, Conflict of Interest: None declared. 